\section{Analysis and Purification of Synthetic Nucleic Acids Using HPLC}

This unit describes a collection of methods and protocols for high-performance liquid chromatography (HPLC) of synthetic nucleic acids (Pingoud et al., 1988; Newton, 1990; Zon, 1990; Warren and Vella, 1993; Oefner and Bonn, 1994; Andrus and Bloch, 1998). For 30 years, HPLC has been a powerful and popular method for the analysis and purification of small molecules, and, more recently, for biopolymers. A considerable industry exists to supply equipment, columns, and technical advice for the practice of HPLC. This unit will emphasize protocols for the analysis and purification of synthetic oligonucleotides. Reversed-phase (see Basic Protocol) and anion-exchange (see Alternate Protocol) are the reigning methods for HPLC of oligonucleotides. Typically anion-exchange gives better resolution and a more predictable elution pattern than reversed-phase. Reversed-phase has advantages in higher capacity purification and the use of volatile buffer systems for easier product recovery. A protocol for detritylating and precipitating oligonucleotides after HPLC purification is also presented (see Support Protocol).

\section{REVERSED-PHASE CHROMATOGRAPHY}

This protocol describes analysis and purification of oligonucleotides under reversedphase conditions. Oligonucleotides undergo hydrophobic interactions with the alkylsilylbonded groups on the reversed-phase adsorbent, resulting in elution patterns that correlate with their overall hydrophobicity. The popular reversed-phase method described in this protocol employs an acetonitrile gradient elution in dilute triethylammonium acetate (TEAA) at neutral $\mathrm{pH}$ on a porous hydrocarbon ( $\mathrm{C} 4$ to $\mathrm{C} 18$ ) silica or polystyrene-divinylbenzene (PS-DVB) adsorbent. The hydrophobicity of triethylammonium, the oligonucleotide counterion, is a significant hydrophobic interactant in the reversed-phase method. Oligonucleotides can be chromatographed tritylated (with the 5' DMTr group attached; DMTr ON) or detritylated (DMTr OFF).

Reversed-phase analysis and purification of tritylated and detritylated oligonucleotides are effective on a column such as Aquapore RP-300 with a moderately hydrophobic, octylsilyl (C8), bonded phase and a large $300 \AA$ average pore size, or a more hydrophobic octadecylsilyl (C18), column, such as the Spheri-5 RP-18, $5 \mu \mathrm{m}$ spherical silica (both available from PE Applied Biosystems). A standard-size column or cartridge $(4.6 \times 220$ $\mathrm{mm}$ ) is suitable for analysis of $\sim 0.3$ to 1.0 OD units of crude mixtures. A maximum of $\sim 10$ OD units can be applied to this size column for purifications. Smaller bore columns2.1-mm, or even microbore, 1.0-mm-offer some advantages in speed and reduced mobile-phase consumption. A larger column $(7 \times 250 \mathrm{~mm}$ or $10 \times 250 \mathrm{~mm})$ gives better separation with larger loadings. To purify the entire product from a $10-\mu$ mole scale synthesis requires many preparative injections.

\section{Materials}

Oligonucleotide sample

Triethylamine

0.1 M triethylammonium acetate, $\mathrm{pH} 7$ (TEAA; PE Applied Biosystems) for dissolving sample

Mobile phase solution A: 0.1 M TEAA, pH 7.0

Mobile phase solution B: acetonitrile
UNIT 10.5

BASIC

PROTOCOL

Purification and Analysis of Synthetic Nucleic Acids and Components

\subsection{1}

Supplement 1 
HPLC instrumentation, capable of both analysis and purification up to about several milligrams oligonucleotide per injection with the following features and specifications:

Injector: autosampler (preferred) or manual syringe

Pumping system: ternary (preferred) or binary, $0.1-5 \mathrm{~mL} / \mathrm{min}$

Detector: UV/fluorescence (preferred) or UV/VIS variable between 190 to

$600 \mathrm{~nm}$

Data: integrating data system (preferred) or chart recorder

Gradient system: displays and stores for redisplay and reformatting (preferred)

or programmable

Column: 4.6 × 220-mm Aquapore RP-300 (Applied Biosystems) or Spheri-5

RP-18 (Applied Biosystems)

Fraction collector

1. Dry the quantity of oligonucleotide needed for analysis (0.05 to 0.1 OD unit) or purification ( $>0.1$ OD unit) in a vessel (e.g., microcentrifuge tube) under vacuum. For tritylated oligonucleotides, add a drop of triethylamine periodically during the evaporation process as a preservative.

To prevent inadvertent detritylation of tritylated oligonucleotides, avoid heat and acid during the drying step. Evaporation of ammonium hydroxide should be done at room temperature when the $5^{\prime}$ trityl is being preserved on the oligonucleotide.

2. Dissolve the sample immediately in sufficient $0.1 \mathrm{M}$ triethylammonium acetate (TEAA) $\mathrm{pH} 7$ for injection.

Typical injection volumes for analysis are 10 to $50 \mu \mathrm{L}$, or $10 \mu \mathrm{l}$ to $2 \mathrm{ml}$ for purification limited by the sample loop size of the injector. Sample loops as large as $2 \mathrm{~mL}$ are available for purification injections.

3. Store tritylated oligonucleotides, if necessary, in the TEAA buffer, adding $\sim 10 \%(\mathrm{v} / \mathrm{v})$ triethylamine as a preservative for tritylated oligonucleotides.

It is difficult or impossible to keep the trityl intact on oligonucleotides upon prolonged storage under any conditions. The trityl group is also unstable if the tritylated oligonucleotide is stored on the solid support, prior to cleavage.

4. Program the gradient system to start with $100 \%$ mobile phase solution A $(0.1 \mathrm{M}$ TEAA), increasing the percentage of mobile phase solution B (acetonitrile) with time (Table 10.5.1). Ensure that sufficient mobile phase has been installed to keep intakes covered during run.

The mobile phase most often used for tritylated and detritylated oligonucleotides is an increasing gradient of acetonitrile in 0.1 M TEAA, pH 7.0 (Table 10.5.1). Most crude

Table 10.5.1 Gradient and Mobile Phase for Reversed-Phase HPLC ${ }^{a}$

\begin{tabular}{cc}
\hline Elapsed time (min) & $\begin{array}{l}\% \text { mobile phase B at } \\
\text { elapsed time }\end{array}$ \\
\hline 0 & 8 \\
24 & 20 \\
34 & 40 \\
\hline
\end{tabular}

${ }^{a}$ Gradient conditions are based on a flow rate of $1 \mathrm{~mL} / \mathrm{min}$ using a Spheri-5 RP-18, $5 \mu \mathrm{m}$ diameter spherical silica, 4.6 $\times 200 \mathrm{~mm}$, or Aquapore RP-300 C-8, $7 \mu \mathrm{m}$ diameter, $300 \AA$ pore, $4.6 \times 220 \mathrm{~mm}$, (all available from PE Applied Biosystems) column at ambient temperature with a $60 \mathrm{~min}$ injection cycle. 
reaction mixtures in the range of 15 to 40 nt can be analyzed and purified using the linear gradient system in Table 10.5.1. This simple gradient is useful for tritylated and detritylated oligonucleotide analysis and purifications.

5. Equilibrate the HPLC system with the starting mobile phase composition until a flat baseline is achieved at the desired detection wavelength.

6. Inject the sample.

7. Collect the desired fractions either with an automated fraction collector, or by observing the chromatogram in real-time and manually collecting the eluate.

Tritylated oligonucleotides will elute at 25 to 35 min and detritylated oligonucleotides at -12 to 15 min under the recommended conditions (Table 10.5.1).

\section{ANION-EXCHANGE HPLC}

This protocol describes analysis and purification of oligonucleotides under anion-exchange conditions. The anion-exchange method consists of a salt-gradient elution on a tertiary or quaternary ammonium-derivatized porous silica or polymeric adsorbent. Denaturing cosolvents such as formamide may be added to minimize hydrogen bonding.

\section{Additional Materials (also see Basic Protocol)}

Mobile phase solutions A and B for anion exchange (see recipe)

Aquapore AX-300 (PE Applied Biosystems) or DNAPac Pa-100 (Dionex) column

1. Dry the quantity of oligonucleotide needed for analysis ( 0.05 to 0.1 OD units) or purification ( $\leq 1$ O.D. units) in a vessel (e.g., microcentrifuge tube) under vacuum. Anion-exchange samples are typically analyzed and purified detritylated.

Crude oligonucleotides in the ammonium hydroxide solution do not have to be dried when using polymeric adsorbent anion-exchange columns.

2. Dissolve the sample in sufficient mobile phase $\mathrm{A}\left(10 \mathrm{mM} \mathrm{NaClO}_{4}\right)$ for injection.

Typical injection volume for analysis is 10 to $50 \mu \mathrm{L}$, or $10 \mu \mathrm{l}$ to $2 \mathrm{ml}$ for purification, limited by the sample loop size of the injector. Sample loops as large as $2 \mathrm{~mL}$ are available for purification injections.

3. Program the gradient system to start with $100 \%$ mobile phase $\mathrm{A}(10 \mathrm{mM} \mathrm{NaClO})_{4}$, increasing the percentage of mobile phase $\mathrm{B}\left(300 \mathrm{mM} \mathrm{NaClO}_{4}\right)$ with time (Table 10.5.2). Ensure that sufficient mobile phase has been installed to keep intakes covered during run.

4. Equilibrate the HPLC system with the starting mobile phase composition until a flat baseline is achieved at the desired detection wavelength.

5. Inject the sample.

6. Collect the desired fractions either with an automated fraction collector, or by observing the chromatogram in real-time and manually collecting the eluate.

Oligonucleotides will elute at 15 to 25 min under the recommended conditions (Table 10.5.2).

Purification and 
SUPPORT PROTOCOL

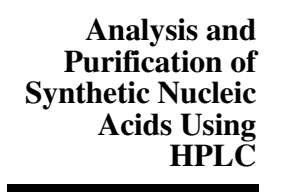

10.5.4

Table 10.5.2 Gradient for Anion-Exchange HPLC ${ }^{a}$

\begin{tabular}{cc}
\hline Elapsed time $(\mathrm{min})$ & $\begin{array}{c}\% \text { mobile phase B at } \\
\text { elapsed time }^{b}\end{array}$ \\
\hline 0 & 0 \\
20 & 70 \\
25 & 70 \\
26 & 0
\end{tabular}

${ }^{a}$ Gradient is based on a $1 \mathrm{~mL} / \mathrm{min}$ flow rate at $25^{\circ} \mathrm{C}$ using an Aquapore AX-300 (PE Applied Biosystems) or DNAPac PA-100 (Dionex) column with a 35 min injection cycle.

${ }^{b}$ Mobile phase solution A is $10 \mathrm{mM} \mathrm{NaClO}_{4}$ and mobile phase solution $\mathrm{B}$ is $300 \mathrm{mM} \mathrm{NaClO}$. See recipe in Reagents and Solutions.

\section{POST-HPLC DETRITYLATION AND PRODUCT ISOLATION}

After collection of the purified tritylated oligonucleotide, the trityl group must be removed. The evaporated sample, which could appear as an oil, is detritylated with acetic acid at room temperature. The acidic treatment does not cause depurination because nucleotides are much more stable to acid after deprotection. Direct precipitation removes acetic acid, the DMTr cation, and byproducts, and ultimately yields the purified oligonucleotide in sodium salt form, dried as a pellet. A final analytical separation will give confirmation of purity.

Post-HPLC isolation is simple for collected fractions of detritylated oligonucleotides by reversed-phase purification because detritylation is not required (see step 2 of this protocol). The combined purified fractions are dried to remove water and the volatile salts (TEAA), and can be used directly in most applications. The purified product can also be ethanol precipitated with concomitant counterion exchange, e.g., sodium for triethylammonium, by the following protocol whereby each OD unit of oligonucleotide is dried and dissolved in $20 \mu \mathrm{L}$ water and carried forward from step 3 (Andrus, 1992).

\section{Materials}

Purified oligonucleotide

$80 \%$ acetic acid

$3 \mathrm{M}$ sodium acetate

Absolute ethanol or 2-propanol

1. Dry the combined, purified oligonucleotide fractions (see Basic Protocol and Alternate Protocol) under vacuum. Incorporate all fractions in a small vessel, such as a 1.5-mL microcentrifuge tube. If the evaporated sample leaves a white film on the bottom on the vial, probably some residual TEAA remains in the sample. Add a small amount $(\sim 100 \mu \mathrm{L})$ of distilled, deionized water and evaporate to remove these salts (several evaporations may be necessary).

2. Dissolve the tritylated oligonucleotide in $20 \mu \mathrm{L} 80 \%$ acetic acid per OD unit, with vortexing, at room temperature. Let stand $20 \mathrm{~min}$.

Omit this step for detritylated samples. Phosphorothioate oligonucleotides may require brief heating at $65^{\circ} \mathrm{C}$.

3. Add $5 \mu \mathrm{L} 3 \mathrm{M}$ sodium acetate and $60 \mu \mathrm{L}$ absolute ethanol per OD unit oligonucleotide and mix by vortexing. For very short oligonucleotides ( $<15$-mers), substitute 2 propanol for ethanol to ensure complete precipitation. 
4. Chill the precipitation at $\leq 4^{\circ} \mathrm{C}$ for $\sim 30 \mathrm{~min}$. Microcentrifuge $5 \mathrm{~min}$ at $\sim 10,000 \times \mathrm{g}$, at ambient temperature or colder.

5. Remove the supernatant with a pipet, micropipet, or by decanting, being careful not to disturb the pellet.

Small quantities $(<100 \mu \mathrm{g})$ may not be visible.

6. Add another $60 \mu \mathrm{L}$ absolute ethanol per OD unit to the pellet, mix briefly, and microcentrifuge 1 to $5 \mathrm{~min}, \sim 10,000 \times \mathrm{g}$, at ambient temperature or colder.

7. Discard the supernatant, being careful not to disturb the pellet. Dry the purified oligonucleotide pellet by vacuum centrifugation or by leaving open on the benchtop.

8. Resuspend the detritylated, desalted oligonucleotide in aqueous medium and quantitate by $A_{260}$.

\section{REAGENTS AND SOLUTIONS}

Use deionized, distilled water in all recipes and protocol steps. For common stock solutions, see APPENDIX 2A; for suppliers, see SUPPLIERS APPENDIX.

\section{Mobile phase solutions $A$ and $B$ for anion exchange}

Mobile phase solution A (10 mM NaClO $\mathrm{mM}$ final; Fluka) in $1 \mathrm{~L}$ distilled or deionized water. Adjust $\mathrm{pH}$ to 8.5 with $0.1 \mathrm{~N}$ $\mathrm{NaOH}$.

Mobile phase solution $\mathrm{B}\left(300 \mathrm{mM} \mathrm{NaClO}\right.$, $\mathrm{pH}$ 8.5): Dissolve $42.1 \mathrm{~g} \mathrm{NaClO}_{4} \cdot \mathrm{H}_{2} \mathrm{O}$ (300 mM final) in $1 \mathrm{~L}$ distilled or deionized water. Adjust $\mathrm{pH}$ to 8.5 with $0.1 \mathrm{~N}$ $\mathrm{NaOH}$.

\section{COMMENTARY}

\section{Background Information}

HPLC embodies the combination of analysis and purification. The analytical chromatogram gives information about (1) purity quantitation, (2) identity by comparison with known samples, and (3) synthesis efficiency. HPLC is a scaleable method, with the largest capacity of any method for oligonucleotide purification. Other aspects of HPLC are a high degree of automation, high resolution, high sensitivity, and ease of pure product recovery. Parameters can be tailored for each separation, as there are many commercial adsorbents (columns and cartridges) for evaluation and purification of nucleic acids. HPLC technology is evolving in the direction of smaller-diameter, nonporous, spherical adsorbent particles packed in smaller columns, with benefits in separation speed, resolution, throughput, and automation.

Because of the resolution limitations of both reversed-phase and anion-exchange HPLC for longer oligonucleotides, HPLC is a useful system for the reliable analysis and purification of oligonucleotides up to $\sim 50 \mathrm{nt}$ in length. Longer oligonucleotides are best analyzed by electro- phoretic methods such as slab gel (PAGE, UNIT 10.4) or gel capillary electrophoresis.

At the current level of synthesizer efficiency and reagent quality, the crude oligonucleotide may be sufficiently pure to function in some experiments. However, careful purification, analysis quantitation, and other preparations are necessary for longer oligonucleotides and for experiments where oligonucleotide purity is critical, especially those requiring cloning and expression. Furthermore, failure to document oligonucleotide purity (e.g., by HPLC) may cloud the interpretation of applications with ambiguous results, as well as the quality control aspects of a laboratory.

Automated, solid-phase, phosphoramidite chemistry (Alul, 1993) gives oligonucleotides with a final average yield per base addition of $\sim 98 \%$, lessening slightly at the larger scales (see Table 10.3.1). At this level of efficiency, synthesis of a 20 nt oligonucleotide will result in $\sim 70 \%$ of the theoretical yield. Depending on the detection method and definition of purity, the sample will also be $\sim 70 \%$ pure. The major side-products are shorter oligonucleotides that failed to couple during synthesis and were ef-
Purification and Analysis of Synthetic Nucleic Acids and Components 
fectively capped to prevent further extension. These failure products typically elute earlier in HPLC than the full length product. Initial analysis by MALDI-TOF (UNIT 10.1) or electrospray mass analysis (UNIT 10.2) may be advised in some labs to ensure the presence of the full-length oligonucleotide.

Other side reactions during synthesis lead to small amounts of later-eluting, more hydrophobic, or highly charged impurities. The yield after HPLC purification is $\sim 50 \%$ of the crude yield (see Table 10.3.1).

Reversed-phase (RP) columns separate by hydrophobic differences. The solid particles within the reversed-phase column are usually silica bonded with hydrocarbon chains via silanol linkages. The polar, aqueous mobile phase and the hydrophobic adsorbent are considered to be in the "reversed" phase, compared to traditional, normal-phase chromatography which uses nonpolar organic solvents and underivatized, polar silica gel. Anion-exchange columns (discussed below) separate oligonucleotides based on charge differences. The particles are typically inorganic silica, derivatized with charged groups such as alkyl ammonium (typically weak or strong anion exchangers). Polymeric particles are also commercially available and useful for reversed-phase and anion-exchange column adsorbents.

\section{Reversed-phase}

Interactions between the oligonucleotide sample (analyte) and the stationary adsorbent packed in the column determine the rate and order of elution. The composition of the liquid mobile phase is typically altered throughout the run (gradient), as opposed to being held constant (isocratic). The mobile phase is aqueous, with dissolved organic solvents or ionic salts, that compete with the sample for interactive adsorbent sites, or otherwise influence elution of the sample. Components of the mobile phase may also act as denaturants in minimizing hydrogen bonding. Stable intramolecular or intermolecular conformations, known as secondary structures, may exist for oligonucleotides with certain sequences.

The large, hydrophobic dimethoxytrityl (DMTr) group may be left on or removed from the $5^{\prime}$ end of the oligonucleotide, according to the option selected by the DNA synthesizer user. Highly efficient synthesis (high coupling yield, minimum side reactions, complete capping and detritylation) gives crude oligonucleotides with only the desired, full-length product bearing a DMTr group. Theoretically, the only oligonucleotide bearing the terminal $5^{\prime}$ DMTr group is the full-length product. All the other sequences that failed to couple in the crude reaction mixture will have been acetylated at the $5^{\prime}$ position during capping. Upon deprotection in ammonia, the failure sequences will have 5'-hydroxyl groups and be separable from the product by a large difference in hydrophobicity due to the presence of the terminal $5^{\prime}$ DMTr group on the full-length product. With the proper gradient and mobile phase conditions, reversed-phase HPLC will easily separate the two types of oligonucleotides. The tritylated product, being more hydrophobic, will be retained longer on the column than the shorter, nontritylated failure sequences and other components of the crude reaction mixture. The retention time differences between tritylated and nontritylated oligonucleotides in the crude oligonucleotide mixture are quite large, especially by the reversed-phase method. Since reversed-phase columns have much greater capacity for tritylated oligonucleotides, this is the preferred mode for purification. However, the trityl group is labile and it can be problematic to preserve it quantitatively on the oligonucleotide during sample preparation or storage. For this reason, and for greater resolution, HPLC of detritylated oligonucleotides is preferred for analysis. Polymeric adsorbents, such as highly cross-linked, rigid polystyrene beads (Ikuta et al., 1984; Germann et al., 1987; Huber et al., 1993), are useful alternatives to hydrocarbon-bonded silica in reversed-phase columns and can use the same gradients and mobile phases (Table 10.5.1). The polymeric columns (Polymer Laboratories, Hamilton, Sarasep, and Rapp Polymere) have the advantages of stability toward high temperature, $\mathrm{pH}$ extremes, and aggressive solvents. They typically exhibit longer lifetimes than their silica counterparts and show very high capacity for tritylated oligonucleotides. High $\mathrm{pH}$ of the mobile phase and heated columns, act as denaturants in the reversed-phase process. These denaturing conditions eliminate some of the secondary structure problems mentioned above.

\section{Anion-exchange}

Anion-exchange HPLC is another efficient technique for analysis and purification (Drager and Regnier, 1985; Maisano et al., 1989; Bergot and Egan, 1992; Huber et al., 1996). Separation is largely based on charge differences. Each oligonucleotide in the crude mixture has a different net charge based on the number of phosphate groups in the molecule (base length) and 
on the respective charges on the bases (Krstulovic, 1987). Therefore, base composition can affect the ion-exchange separation process. Resolution is excellent in the analysis and purification of small quantities of detritylated oligonucleotides. Single-base resolution can usually be achieved for oligonucleotides up to 25 $\mathrm{nt}$ in length and sometimes longer, depending on the sequence. The advantages of anion-exchange are ease of sample preparation, without the need to preserve the trityl group, and recovery of the purified compound in a ready-to-use form. The detritylated oligonucleotide sample can be redissolved in deionized water and stored in a refrigerator or freezer indefinitely. Anion-exchange polymeric adsorbents allow for high-pH mobile phase analysis, which neutralizes nucleobases and denatures secondary structures. Separation of the crude mixture is accomplished by slowly increasing the ionic strength (concentration) of the mobile phase (Table 10.5.2). The longer, more highly charged, oligonucleotides will thus elute later than shorter ones, with the product peak eluting last since it is the most highly charged.

Phosphate groups are occasionally masked by secondary structures and will not exhibit the effect of a full charge, causing deviation from the predictable size-dependent elution pattern. Oligonucleotides have a hydrophilic, polyanionic backbone decorated with hydrophobic and hydrogen-bonding nucleobases, capable of complex interactions with the adsorbent. Stable intra or intermolecular conformations, secondary structures from Watson/Crick base-pairing or from Hoogsteen base-pairing in G-rich sequences can result in complex chromatograms and variable retention times.(Kang et al., 1992; Wang, 1993). The secondary-structure effects can be minimized by adding a strong denaturant, such as formamide, to the mobile phase, or by heating the column up to $\sim 70^{\circ} \mathrm{C}$. Alternatively, in the case of anion exchange HPLC, alkaline mobile phases ( $\mathrm{pH} 12$ ) can be employed. Resolution decreases as larger oligonucleotides are eluted, because the relative difference in charge between longer oligonucleotides is less than in the shorter size ranges. Also, there is inevitable peak broadening as a function of increasing oligonucleotide length. The retention times of oligonucleotides on ionexchange HPLC, using identical mobile phase conditions, will usually be quite reproducible and predictable. Therefore, sequence length can be confirmed with reliability. Crude oligonucleotides may be injected ( 0.02 to $0.2 \mathrm{OD}$ units per run) directly onto polymeric columns from ammonium hydroxide solution (Ausserer and Biros, 1995).

\section{Labeled oligonucleotides, RNA, and analogs}

Covalent attachment of molecules, such as fluorescent dyes, biotin, proteins, and others, can be made at virtually any site on the oligonucleotide (Goodchild, 1990; Alul, 1993; Andrus, 1995). These include the $5^{\prime}$ and $3^{\prime}$ ends, through the bases, and through an internucleotide phosphate modification. HPLC of internucleotide analog DNA, such as phosphorothioate, methylphosphonate, phosphorodithioate, and phosphoramidate, has been investigated (Zon 1993; Andrus and Bloch, 1998).

Analysis and purification by HPLC are feasible for most of these derivatized oligonucleotides, with only slight modifications to the methods. Many of these analogs are very hydrophobic and require higher organic modifier concentration in the mobile phase for reversedphase separations. Some analog DNAs have chiral phosphorus atoms, leading to complex diastereomeric mixtures. The retention time and elution pattern of conjugated oligonucleotides will be affected by the hydrophobicity of the attached species. For example, biotin, especially with a long linking chain, imparts significant additional hydrophobicity and increases the retention time.

Synthetic oligoribonucleotides (RNA) are well-behaved HPLC analytes (Sproat et al., 1995). Extra care is warranted for analysis and purification since the synthesis efficiency is lower, reagent costs are higher, and most of the biological applications for RNA are more purity-critical than for DNA. The standard 2'-Otert-butyldimethylsilyl protecting group of oligoribonucleotides is usually removed with tetrabutylammonium fluoride in the final deprotection operation and requires careful separation of the copious tetrabutylammonium salts from the RNA. New reagents and post-synthesis methods have been developed to address this problem (Applied Biosystems, 1995). RNA is more likely to exhibit secondary-structure effects than DNA, especially those sequences designed with intramolecular hydrogen-bonding, e.g., ribozymes (Noller, 1984; Symons, 1992). Sometimes heating the column in an oven gives a better indication of purity due to denaturation of secondary structures and the resulting simplification of the chromatogram. RNA is more hydrophilic than DNA, which decreases the loading capacity on HPLC columns.
Purification and Analysis of Synthetic Nucleic Acids and Components 


\section{Critical Parameters and \\ Troubleshooting}

HPLC is meant to be an automated, largely unattended operation. However, the hardware is relatively complex and expensive, compared to PAGE (polyacrylamide gel electrophoresis, see UNIT 10.4). A minimum of down time and consistent, optimal results can be attained by observing the following proven HPLC maintenance operations:

1. Sample preparation: Filter or centrifuge to remove insoluble particulate matter.

2. Pump maintenance: Clear all salts and buffers from the pumps at the end of a session. Mobile phases should be maintained under a positive pressure of $\sim 5$ psi of inert gas, e.g., helium, to prevent bubble formation and evaporation.

3. Data storage: Store chromatograms in personal computer-based systems.

4. Mobile phases: Filter and degas.

5. Guard columns: Mount in line between the injector and the column. A guard column can extend the column lifetime by trapping insoluble impurities and chemically damaging species in the sample.

Manufacturers of HPLC equipment often provide useful literature and references for learning to use and maintain the system, as well as for specific applications. In configuring an HPLC system, it is imperative that extra column dead volumes be minimized, especially for analytical-scale and microbore HPLC. Most systems are configured primarily for analysis; therefore dead volumes and mixing points are often minimized only up to the flow cell of the detector. For preparative work, dead volumes and mixing points must be eliminated between the flow cell and point of collection. Nucleic acids-DNA and RNA — absorb light strongly at $\sim 260 \mathrm{~nm}$ due to their chromophoric, conjugated pyrimidine and purine ring systems. Detecting and quantitating the absorbance of the ultraviolet light can be done precisely. A finite period of time is required for a compound to pass from the detector to the collection port. This time interval can be determined by injecting any UV-absorbing compound, collecting the fractions at specific time points, and measuring the absorbance of the fractions individually. The time corresponding to the fraction containing the strongest UV absorption minus the time of the peak maximum on the chart recorder will be the time lag before samples should be collected. It is important that this time interval be kept under 10 to $20 \mathrm{sec}$ for most accurate preparative work. It is also very help- ful to have a digital readout of the absorbance on a real-time basis. This can be extremely important in the purification of complex mixtures where the points of collection play a key role in obtaining pure material.

The HPLC purification process is different from the analysis process in the following ways. Typically a larger column and higher flow rates are employed for isolating milligram amounts of oligonucleotides. The resolution requirements for purification are not as strict as in analysis. Because the amount of product needed for most applications is quite small, only a portion of the peak need be collected. Since most molecular biology applications require only picomole quantities of pure oligonucleotide, it is better to be more conservative in the collection process and get less quantity of the pure product than to try for a larger amount of less pure product. Hence, good collection techniques and post-purification analysis can routinely produce pure product even when the analytical chromatogram shows a complex mixture.

\section{Anticipated Results}

For conventional UV absorbance detection at or near $260 \mathrm{~nm}$, ng to $\mu \mathrm{g}$ quantities of nucleic acid in 0.1 to $1 \mathrm{~mL}$ peak volumes give signals between the state-of-the-art detection limit $\left(10^{-5} \mathrm{AU}\right)$ and the 1 to 2 AUFS (absorbance units full scale) upper bound of detector linear dynamic range. For milligram-scale separations, detection may be set off the absorbance maximum for DNA at 280 to $300 \mathrm{~nm}$ to keep the plot on scale. Analytical-scale, 2.1 to 4.6 mm i.d. (non-microbore) HPLC columns show good resolution and recovery of ng to mg quantities of nucleic acid. Larger-scale separations for industrial manufacture of ribozyme or antisense therapeutics will require significant scale-up, a topic beyond the scope of this unit.

A typical tritylated oligonucleotide chromatogram is shown in Figure 10.5.1. The initial peak at 5 min retention time is benzamide, the by-product from deprotection of the deoxycytidine and deoxyadenosine bases. Isobutyramide, from the deoxyguanosine base, does not have a chromophore and will not appear in the chromatogram, with the usual UV absorbance detection at $\sim 260 \mathrm{~nm}$. The next group of peaks between 14 and 18 min are the detritylated (tritylOFF), failure sequences. Any oligonucleotide that has accidentally lost its trityl group will also appear in this region. The major peak at $30 \mathrm{~min}$ is the tritylated (trityl $\mathrm{ON}$ ) product. The number of peaks in the region 


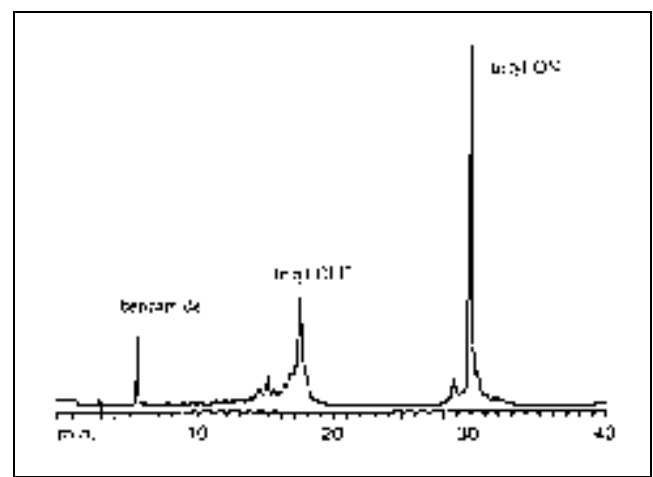

Figure 10.5.1 Chromatogram of reversedphase analysis of tritylated 18-nt oligodeoxyribonucleotide, 5'-DMTr-TCACAGTCTGATCTCGAT-3', using an Aquapore RP-300 column.

where trityl-bearing compounds elute indicates heterogeneity in tritylated species due to the imperfections of DNA synthesis. Longer tritylated oligonucleotides have less overall hydrophobicity.

A typical chromatogram of a detritylated oligonucleotide is shown in Figure 10.5.2. The major peak at $14.5 \mathrm{~min}$ is the 20 -nt product. This peak is surrounded by minor peaks which constitute the various failure sequences and are eluted earlier than shorter tritylated oligonucleotides due to increased charge and less net hydrophobicity. It is hard to identify peaks corresponding to by-products of DNA synthesis from relative retention time because of the

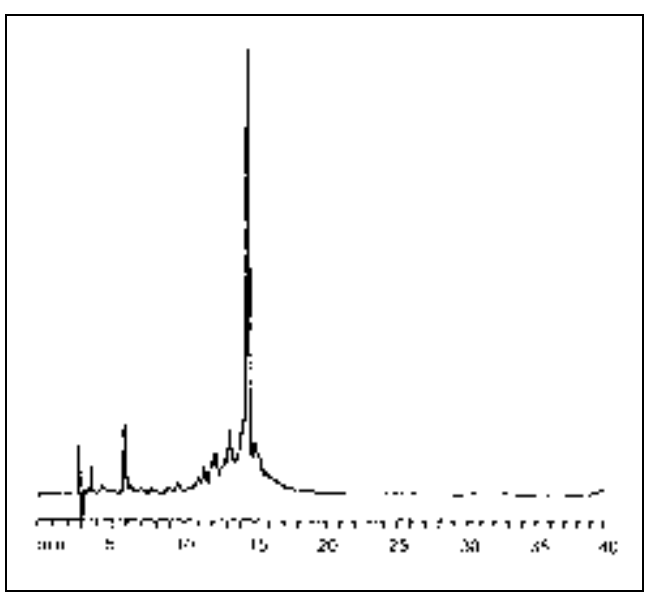

Figure 10.5.2 Chromatogram of reversedphase analysis of detritylated 20-nt oligodeoxyribonucleotide, 5'-CGAGTACTCCAA AACTAATC$3^{\prime}$, using an Aquapore RP-300 column.

complex chromatographic effect of base composition. Oligonucleotides of shorter length than the product are usually eluted earlier than the product. Benzamide appears here at 5.9 min. Detection at 0.01 to 0.1 AUFS is typical for routine examination of the chromatogram (Fig. 10.5.1 and 10.5.2).

Figure 10.5.3 shows a crude 29-nt oligonucleotide synthesized with low efficiency, $96.7 \%$ final average step-wise yield, and analyzed by anion-exchange HPLC (see Alternate Protocol) with the gradient of Table 10.5.2. The failure sequences are well resolved and separated from

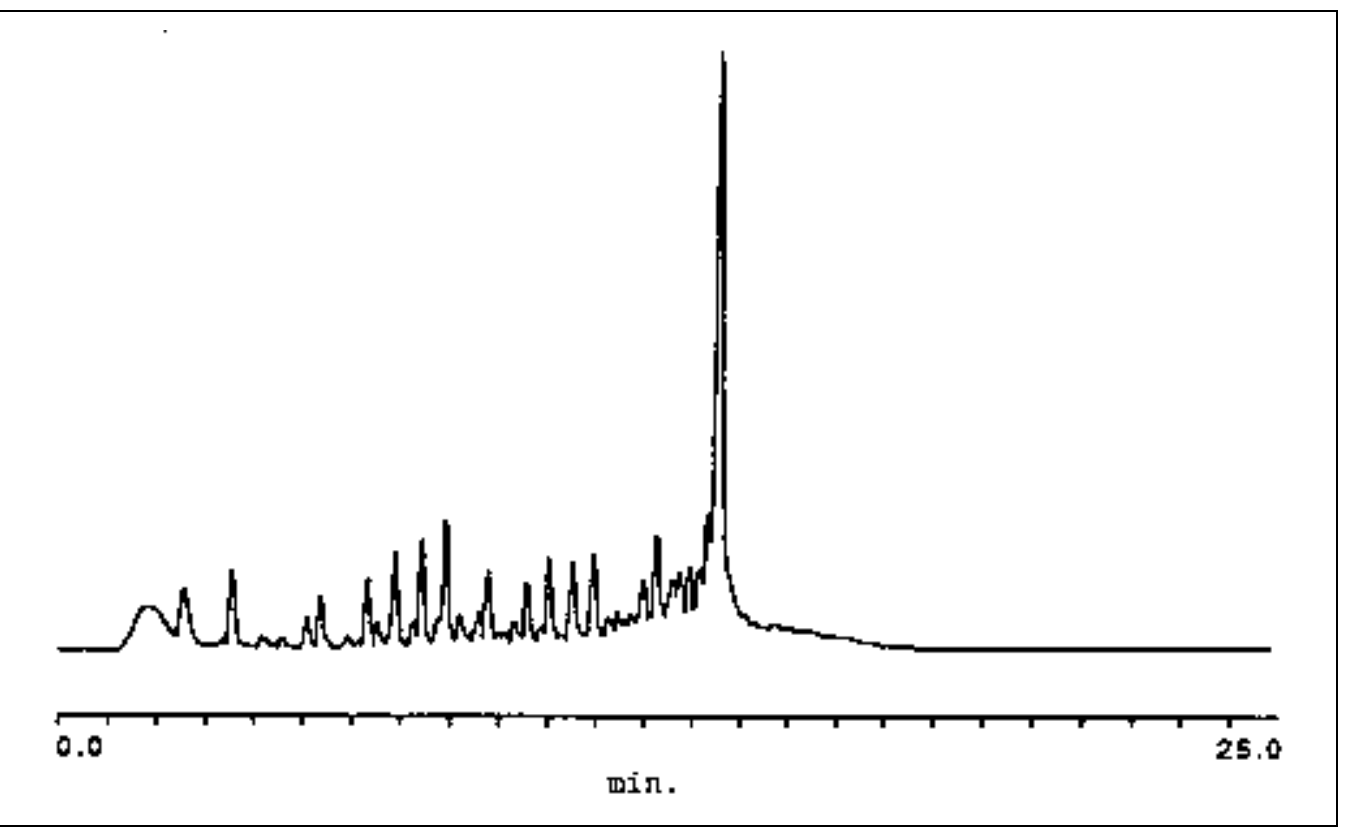

Figure 10.5.3 Chromatogram of anion-exchange analysis of 29-nt oligodeoxyribonucleotide, 5'-CCATGAAGCTTTGACCATGAAAATGGAGA-3', using a DNAPac PA-100 column.

Purification and Analysis of Synthetic Nucleic Acids and Components

\subsection{9}


the product. Resolution by this system is excellent out to at least 50 bases. Sample carryover from one chromatogram to the next may occur due to buildup of sample and other hydrophobic impurities, but treatment with 50\% acetonitrile will help clean the column. Oligonucleotides purified by ion-exchange HPLC must be desalted since the amount of nonvolatile salts collected with the oligonucleotide product will be substantial. Desalting can be achieved by the procedures in UNIT 10.7.

Figure 10.5.4 shows an 18-nt oligonucleotide conjugated with biotin through the $5^{\prime}$ end using the cartridge and gradient of Table 10.5.1 (see Basic Protocol). The biotinylated product elutes at $15.2 \mathrm{~min}$. A small amount of $5^{\prime}$-OH oligonucleotide is present as the smaller preceding peak at $12.9 \mathrm{~min}$. Benzamide is present at $5.9 \mathrm{~min}$.

Fluorescent dyes, most commonly attached through the $5^{\prime}$ terminus, also impart additional hydrophobicity to the oligonucleotide (Oefner et al., 1994). The dye often is charged, either positive or negative. Like biotinylated oligonucleotides, fluorescent dye-labeled oligonucleotides elute later than their $5^{\prime} \mathrm{OH}$ counter- parts. Fluorescence detection is a powerful aid to analysis. Figure 10.5.5 shows a crude $5^{\prime}$ fluorescent dye 20-nt, labeled with 6-carboxyhexachlorofluorescein oligodeoxyribonucleotide phosphoramidite-HEX (Theisen et al., 1992), eluting at 26 min using the Aquapore RP-300 column and the gradient of Table 10.5.1 (see Basic Protocol). A small amount of unlabeled $20 \mathrm{nt}$ is visible at $21 \mathrm{~min}$, well separated from the product.

\section{Purification of oligonucleotides}

Reversed-phase HPLC of tritylated oligonucleotides is the method with the largest capacity for general oligonucleotide purification and is also the most easily scaled-up (Huang and Krugh, 1990). A large cartridge or column (PRP-1, $10 \times 250 \mathrm{~mm}, 10 \mu \mathrm{m}$ diameter; Hamilton) at a flow rate of $4.5 \mathrm{~mL} / \mathrm{min}$ can purify the entire product, $\sim 20-30 \mathrm{mg}$, from a $10 \mu \mathrm{mol}-$ scale synthesis, albeit with considerable loss of resolution relative to analytical-scale HPLC. The collection process during purification is important, since impurities can elute both before and after the product peak. Any unwanted tritylated peaks will usually elute later in the
Analysis and Purification of Synthetic Nucleic Acids Using HPLC

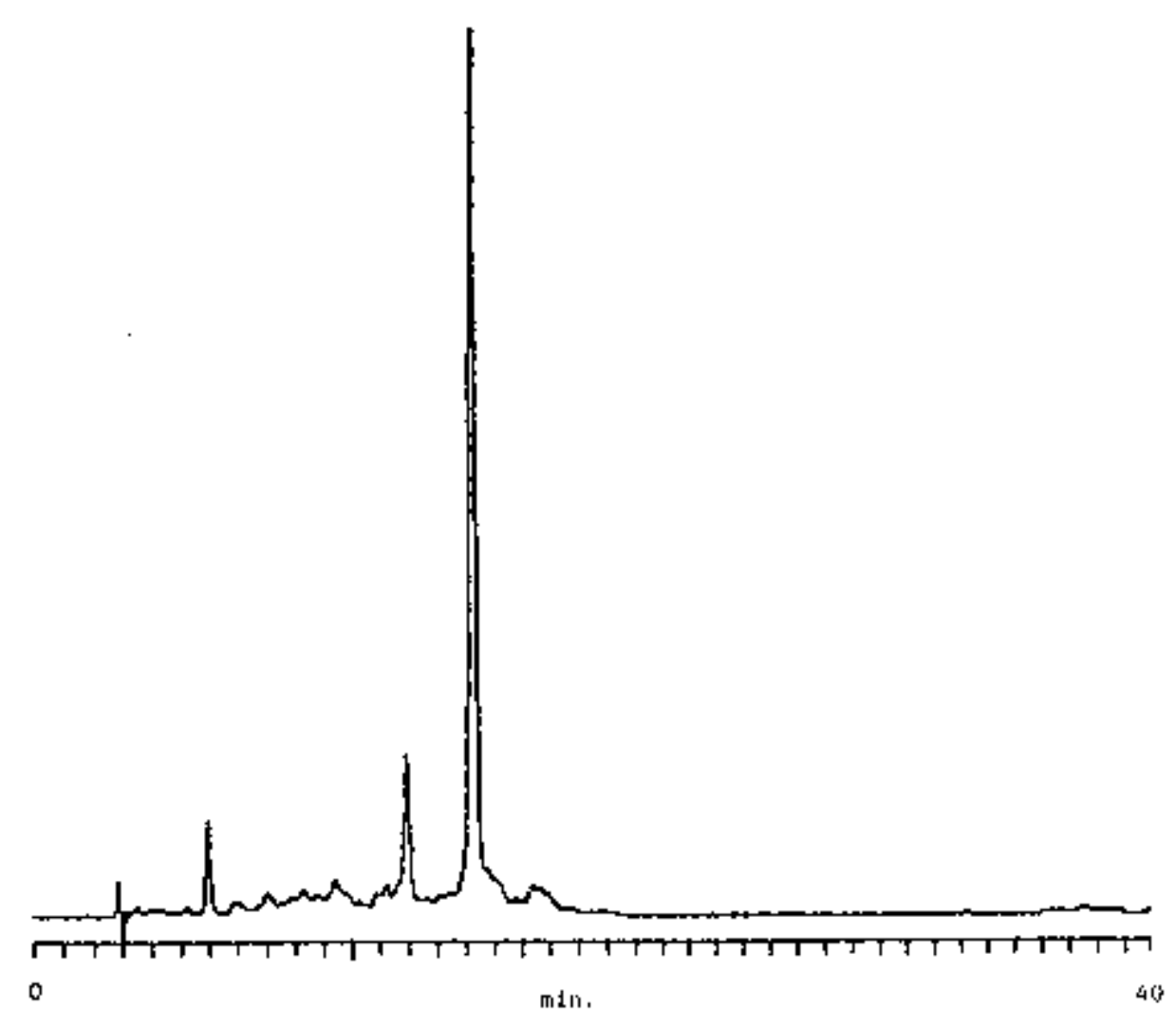

Figure 10.5.4 Chromatogram of reversed-phase analysis of oligodeoxyribonucleotide 5'-biotinTCACAGTCTGATCTCGAT-3', using an Aquapore RP-300 C-8 column. 


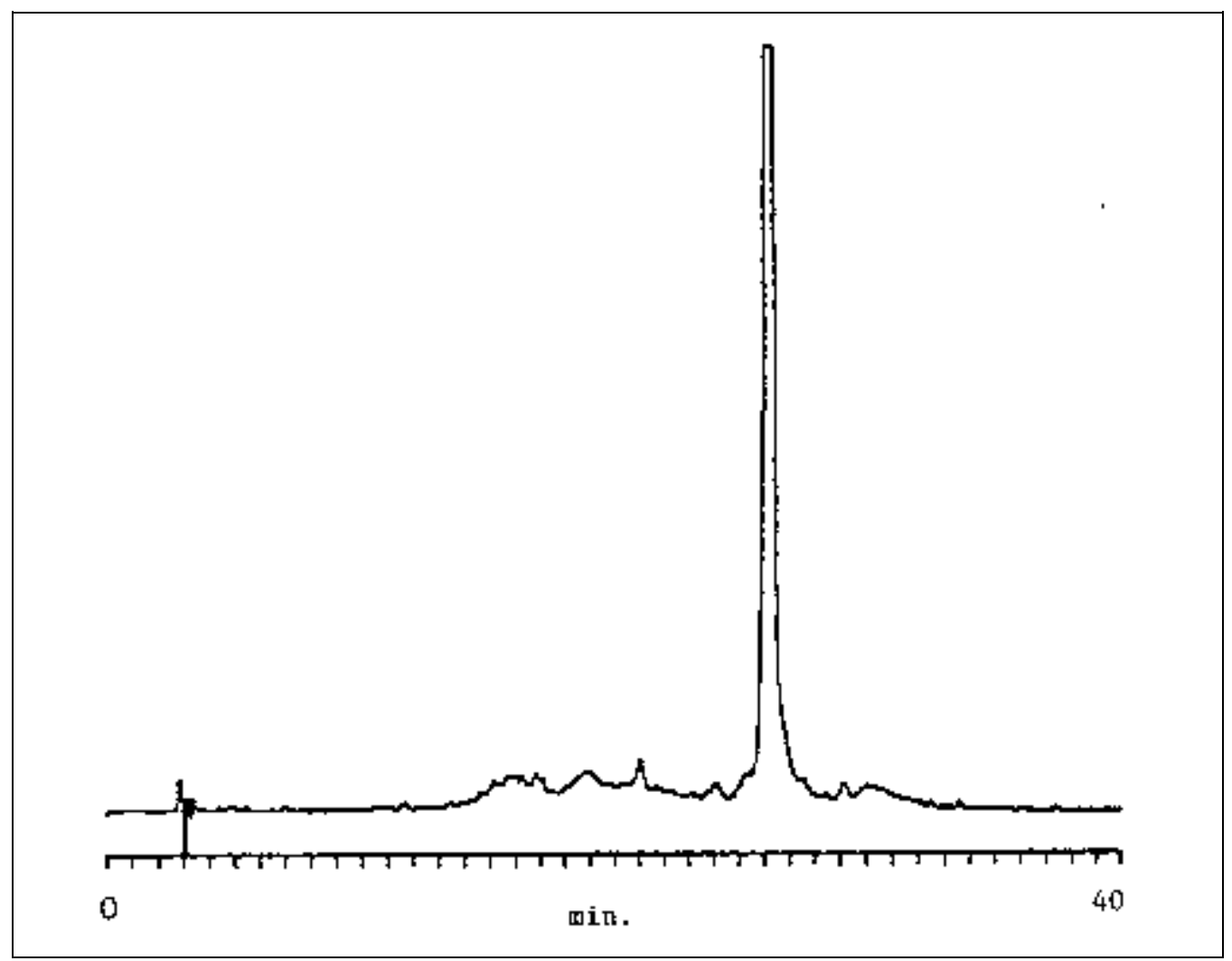

Figure 10.5.5 Figure 10.5.5 Chromatogram of reversed-phase analysis of 5'-HEXACATCTCCCCTACCGCTATA-3', using an Aquapore RP-300 C-8 column.

chromatogram, appearing as separate peaks or as shoulders of the main peak. The collection should start at $50 \%$ of the peak maximum on the upside of the peak to $50 \%$ of the peak maximum on the downside. The preparative chromatogram in Figure 10.5.6 was performed at $290 \mathrm{~nm}$, far off the absorbance maxima of $\sim 260 \mathrm{~nm}$, to allow for better visualization of the resolution under such overloaded conditions (Table 10.5.1). In the chromatogram, benzamide elutes at $6 \mathrm{~min}$, followed by an off-scale broad absorbance by trityl-off failure sequences at 11 to $16 \mathrm{~min}$. The tritylated $22 \mathrm{nt}$ product elutes as an off-scale broad peak from

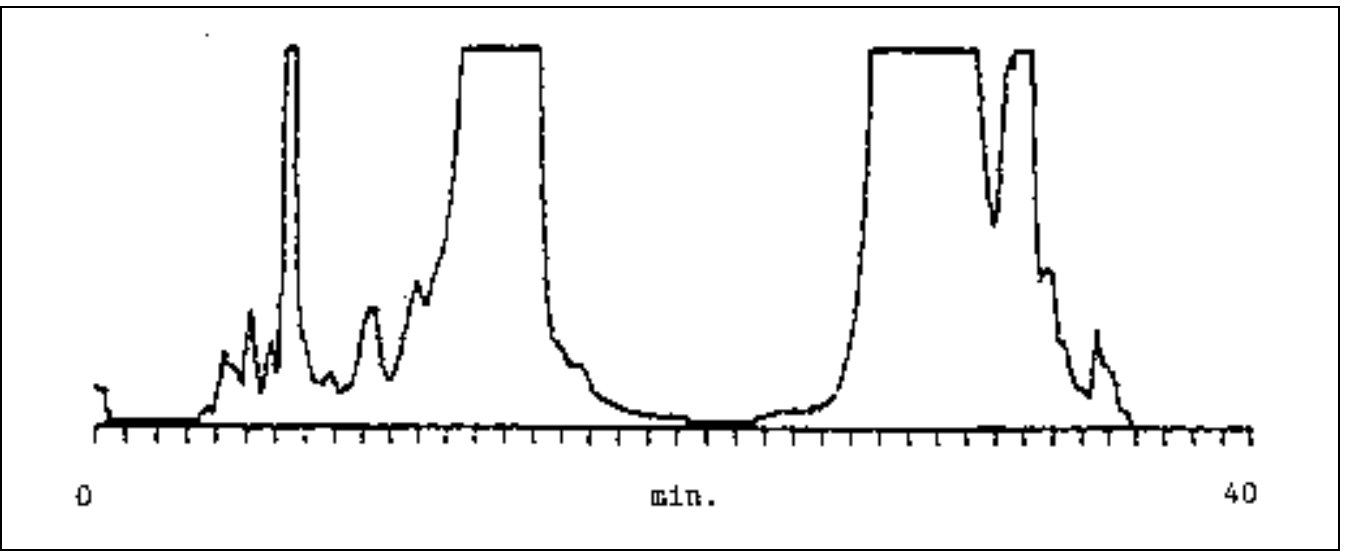

Figure 10.5.6 Chromatogram of reversed-phase preparative purification of $100 \mathrm{OD}$ units crude tritylated 22-nt oligodeoxyribonucleotide, 5'-DMTr-GAATCACAGTCTGATCTCGATT-3', using an Aquapore RP-300, $10 \times 250-\mathrm{mm}, 20 \mu \mathrm{m}$ particle diameter $(\mathrm{ABI})$, a flow rate of $4.5 \mathrm{~mL} / \mathrm{min}$, and detection at $290 \mathrm{~nm}$.

Purification and Analysis of Synthetic Nucleic Acids and Components

10.5.11 
26 to $31 \mathrm{~min}$. The peak from 31 to $33 \mathrm{~min}$ is a set of tritylated failure sequences, more hydrophobic than the product since they are shorter.

\section{Time Considerations}

Most HPLC runs can be conducted in less than $1 \mathrm{hr}$, including time to reequilibrate the mobile phase and conduct the attendant maintenance operations. With an autosampler, HPLC analysis can be conducted virtually around the clock. "Smart" fraction collectors can automatically collect the purified product.

\section{Literature Cited}

Alul, R. 1993. Chemical synthesis of DNA and DNA analogs. In DNA Probes, 2nd ed. (G. Keller and M. Manak, eds.) pp. 69-136. Stockton Press, New York.

Andrus, A. 1992. Evaluating and Isolating Synthetic Oligodeoxynucleotides. Applied Biosystems, Foster City, Calif. Available upon request.

Andrus, A. 1995. Chemical methods for $5^{\prime}$ non-isotopic labelling of PCR probes and primers. In PCR II: A Practical Approach (M. McPherson, B. Hames, and G. Taylor, eds.) pp. 39-54. Oxford University Press, Oxford.

Andrus, A. and Bloch, W. 1998. HPLC of oligonucleotides and polynucleotides. In HPLC of Macromolecules: A Practical Approach (R.W.A. Oliver, ed.) pp. 141-70, Oxford University Press, Oxford.

Applied Biosystems. 1995. Improved 1- $\mu$ mol RNA synthesis, analysis and purification. User Bulletin No. 91

Ausserer, W. and Biros, M. 1995. High-resolution analysis and purification of synthetic oligonucleotides with strong anion-exchange HPLC. Biotechniques 19:136-39.

Bergot, B.J. and Egan, W. 1992. Separation of synthetic phosphorothioate oligodeoxynucleotides from their oxygenated (phosphodiester) defect species by strong-anion-exchange high-performance liquid chromatography. J. Chromatogr. 599:35-42.

Drager, R.R. and Regnier, F.E. 1985. High-performance anion-exchange chromatography of oligonucleotides. Anal. Biochem. 45:47-56.

Germann, M.W., Pon, R.T., and van de Sande. H. 1987. A general method for the purification of synthetic oligodeoxyribonucleotides containing strong secondary structure by reversed-phase high-performance liquid chromatography on PRP-1 resin. Anal. Biochem. 165:399-405.

Goodchild, J. 1990. Conjugates of oligonucleotides and modified oligonucleotides: A review of their synthesis and properties. Bioconjugate Chem. 1:165-187.
Huang, G. and Krugh, T.R. 1990. Large-scale purification of synthetic oligonucleotides and carcinogen-modified oligodeoxynucleotides on a reverse-phase (PRP-1) column. Anal. Biochem. 190:21-25.

Huber, C.G., Oefner, P.J., and Bonn, G.K. 1993. High-resolution liquid chromatography of oligonucleotides on nonporous alkylated styrenedivinylbenzene copolymers. Anal. Biochem. 212:351-358

Huber, C., Stimpf, E., Oefner, P., and Bonn, G. 1996. A comparison of micropellicular anion-exchange and reversed-phase stationary phases for HPLC analysis of oligonucleotides. $L C / G C$ 14:114-127.

Ikuta, S., Chattopadhyaya, R., and Dickerson, R.E. 1984. Reverse-phase polystyrene column for purification and analysis of DNA oligomers. Anal. Chem. 56:2253-2256.

Kang, C., Zhang, X., Ratliff, R., Moyzis, R., and Rich, A. 1992. Crystal structure of four-stranded Oxytricha telomeric DNA. Nature 356:126.

Krstulovic, A. 1987. Nucleic acids and related compounds. In Handbook of Chromatography, Vol. 1, Parts A and B (G. Zweig and J. Sherma, ed.) pp. 161-169; 35-64. CRC Press, Boca Raton, Fla.

Maisano, F., Parente, D., Velati Bellini, A., Carrera, P., Zamai, M., and Grandi, G. 1989. A rapid and efficient method for the purification of synthetic oligonucleotides by high performance anion-exchange chromatography in volatile buffer. Biochromatography 4:279-281.

Newton, P. 1990. Complex biological matrices: Column capacity and separation strategy. $L C / G C$ 8:116-122.

Noller, H.F. 1984. Structure of ribosomal RNA Annu. Rev. Biochem. 53:119-162.

Oefner, P. and Bonn, G. 1994. High-resolution liquid chromatography of nucleic acids. Am. Lab. June 28C-28J.

Oefner, P.J., Huber, C.G., Umlauft, F., Berti, G-N., Stimpfl, E., and Bonn, G.K. 1994. High-resolution liquid chromatography of fluorescent dyelabeled nucleic acids. Anal. Biochem. 223:39-46.

Pingoud, A., Fliess, A., and Pingoud, V. 1988. HPLC of oligonucleotides. In HPLC of Macromolecules (R.W.A. Oliver, ed.) pp. 183. IRL Press, New York.

Sproat, B., Colonna, F., Mullah, B., Tsou, D., Andrus, A., Hampel, A., and Vinayak, R. 1995. An efficient method for the isolation and purification of oligoribonucleotides. Nucleosides Nucleotides 14:255-273.

Symons, R.H. 1992. Small catalytic RNAs. Ann Rev. Biochem. 61:641-671.

Theisen, P., McCollum, C,. and Andrus, A. 1992. Fluorescent dye phosphoramidite labelling of oligonucleotides. Nucl. Acid Symp. Ser. No. 27, pp. 99-100.
Analysis and

Purification of

Synthetic Nucleic

Acids Using HPLC 
Warren, W.J. and Vella, G. 1993. Analysis and purification of synthetic oligonucleotides by highperformance liquid chromatography. In Protocols for Oligonucleotide Conjugates (S. Agrawal, ed.) pp. 233-64. Humana Press, Totowa, N.J.

Wang, K.Y., McCurdy, S., Shea, R.G., Swaminathan, S., and Bocton, P.H. 1993. A DNA aptamer which binds to and inhibits thrombin exhibits a new structural motif for DNA. Biochemistry 32:1899-1904.

Zon, G. 1990. Purification of synthetic oligodeoxyribonucleotides. In High Performance Liquid Chromatography in Biotechnology (W.S. Hancock, ed.) pp. 301. John Wiley \& Sons, New York.
Zon, G. 1993. Oligonucleoside phosphorothioates In Protocols for Oligonucleotides and Analogs (S. Agrawal, ed.) pp. 165-189. Humana Press, Totowa, N.J.

Contributed by Alex Andrus

PE Applied Biosystems

Foster City, California

Robert G. Kuimelis

Phylos, Inc.

Lexington, Massachusetts
Purification and

Analysis of

Synthetic Nucleic

Acids and

Components 